\title{
An Error Control Scheme for Multicast Video Streaming on the Last Hop Wireless LANs ${ }^{\star}$
}

\author{
Junghoon Lee ${ }^{1}$, Mikyung Kang ${ }^{1}$, Gyungleen Park ${ }^{1}$, \\ Hanil Kim ${ }^{2}$, Choelmin $\mathrm{Kim}^{2}$, and Seongbaeg Kim ${ }^{2}$ \\ 1 Dept. of Computer Science and Statistics, \\ 2 Dept. of Computer Education, \\ Cheju National University, 690-756, Jeju Do, Republic of Korea \\ \{jhlee, mkkang, glpark, hikim, cmkim, sbkim\}@cheju.ac.kr
}

\begin{abstract}
This paper proposes and analyzes the performance of an error control scheme for multicast video streaming over IEEE 802.11 WLAN. The proposed scheme makes all packets include the field indicating the number of packets of the message to which they belong, the receiver nodes report errors in a best-effort manner through contention period, and finally access point retransmits the requested packets through the overallocated slot that is unavoidably brought by QoS guarantee. Simulation results show that the proposed scheme can not only efficiently utilize the network bandwidth by reusing the wasted bandwidth for error control but also reduce the deadline miss ratio by $23 \%$ compared with fixed length-based error control scheme without affecting other streams.
\end{abstract}

\section{Introduction}

The great success of IEEE 802.11 technology for WLANs (Wireless Local Area Networks) is creating new opportunities for the deployment of advanced multimedia services such as video conferencing, video multicast, and so on 1 . Audio/video streaming will be a critical part of the wireless communication, as multicast streaming offers an efficient paradigm for transmitting video from a sender to a group of receivers using mobile devices such as PDA, telematics, and so on, with lower network and end-system costs. Unlike the general data traffic, video traffic is delay-sensitive and somewhat tolerant to packet loss through the use of error concealment technique at the video decoder. However, the use of WLANs for the transport of video accompanies some problems resulted from the strict delay constraints of the video traffic and the inherent unpredictability of the wireless link 2 .

The streaming service on WLAN requires a certain type of QoS (Quality of Service) guarantee and run-time error control to provide an acceptable video quality. QoS guarantee means a reservation of resources that can meet the delay

\footnotetext{
* This research was supported by the MIC, Korea, under the ITRC support program supervised by the IITA.
} 
constraint of video stream 3]. The guarantee mechanism inevitably depends on the underlying MAC (Medium Access Control) layer and IEEE 802.11 WLAN standard consists of a basic DCF (Distributed Coordination Function) and an optional PCF (Point Coordination Function) 4]. Due to packet collisions intrinsic to CSMA/CA (Carrier Sense Multiple Access with Collision Avoidance), DCF is not appropriate for video streaming. The QoS guarantee cannot be provided without developing a deterministic access schedule on top of collision-free PCF [5]. However, DCF cannot be totally excluded from WLAN operation because the standard demands that the DCF should be at least long enough to transmit one maximum data unit, to prevent starvation of stations that are not allowed to send during the PCF.

Meanwhile, lost packets constitute one of the main causes of video quality degradation, while the wireless channel error is characterized as bursty and location dependent [6]. Due to the delay constraints, the number of retransmission that can be used is limited and usually small. In case of multiple clients, each client will have different channel conditions, processing capabilities, and only limited feedback channel capabilities. The error control scheme for multicast video is not aiming at recovering all lost packets but recovering as many packets as possible. Most importantly, the error control procedure should not affect the other guaranteed traffics in WLAN.

The real-time guarantee inevitably generates overallocation during PCF as the time constraints of message stream are usually described with the maximum value of message size. Retransmission via this overbooked bandwidth does not interfere the transmission of other guaranteed messages. In the other hand, if we let error report containing retransmission request be delivered via the DCF period in a best-effort manner, the entire error control procedure can be carried out without any influence to other real-time messages. Finally, though the variable message size makes it hard to decide when to report the error list, the receivers can determine the completion of message transmission by counting Beacon frame AP (Access Point) generates periodically in WLAN. Based on the requirements described previously, this paper will propose and analyze the performance of an error control scheme for multicast video stream on IEEE 802.11 WLAN. We focus on the video streaming scenario in the last mile network, namely, between $\mathrm{AP}$ and the mobile devices.

The rest of this paper is organized as follows: After reviewing some related works in Section 2, Section 3 describes the background of this paper including network, error, and message models. Section 4 proposes an error control scheme and Section 5 shows the result of performance measurement. Finally, Section 6 summarizes and concludes this paper.

\section{Related Works}

Error control in wireless network has been intensively studied for both unicast and multicast. Most of the approaches use ARQ (Automatic Retransmission reQuest), FEC (Forward Error Correction), or a combination of both 7]. Pure 
ARQ-based schemes are less appropriate for the multicast case due to ACK explosions and the requirement to retransmit different packets to the respective users. For significant packet loss rates, each user will require frequent packet replacement, and different receivers are most likely to require different packets. To respond to requests by multiple users, we may have to resend a significant fraction of the original data even for small loss rates. For a packet network with dynamic bandwidth, a different class of the source coding technique called progressive coding is better suited. However, this method has the problem in deciding optimal coding parameter on wireless channel.

As a hybrid ARQ mechanism, Majumdar et al. have proposed a method that combines the reliability and fixed delay advantage of forward error control coding with bandwidth-conserving channel-adaptive properties of ARQ protocol[2]. Masala has also proposed a multicast scheme that aims at globally optimizing the parameters involved in a real-time video transmission, ranging from video encoding and packetization to the 802.11 MAC interface parameters [3]. These schemes are mainly built on top of DCF, so they didn't consider the effect of QoS reservation.

$\mathrm{Lu}$ et al. have proposed a timestamp-based content-aware adaptive retry mechanism where MAC dynamically determines whether to send or discard a packet by its retransmission deadline, which is assigned to each packet according to its temporal relationship and error propagation characteristics with respect to other video packets within the same group of pictures [7]. However, their scheme is too complex to be exploited in the WLAN standard, as it crosses the protocol layer boundaries.

\section{Background}

\subsection{Network Model}

We consider a wireless-cum-wired network scenario as shown in Fig. 18]. A fixed node is connected with an AP through a wired link which is overprovisioned so that no packets are dropped at its end. This model enables us to concentrate on the behavior of inside WLAN part as it assumes ideal environment of no packet loss or jitter for outside WLAN part. Each cell is assumed to consist of an AP and multiple MSs (Mobile Stations), while each flow is either uplink (from MS

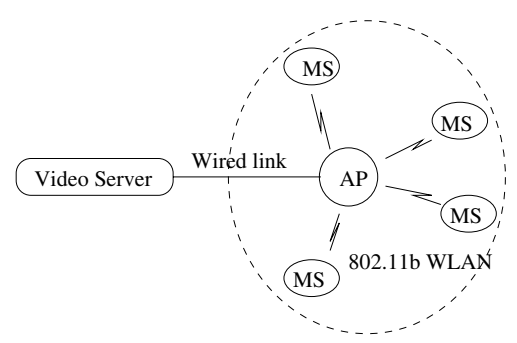

Fig. 1. Network model 


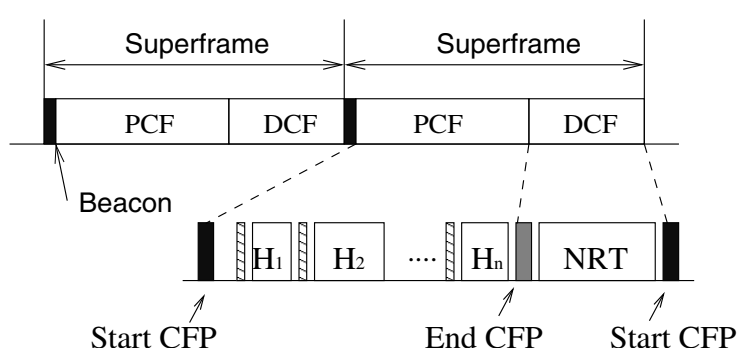

Fig. 2. Time axis of wireless LAN

to AP) or downlink (from AP to MS). Most flows are downlink, and the stream flow arrives periodically from the remote server outside the cell via reliable wired link. The multicast on WLAN prevents the automatic ACK transmission from the receiver.

The IEEE 802.11 was developed as a MAC standard for WLAN 4]. The standard divides the time axis into the series of superframes, and each of them consists of CFP (Contention Free Period) and CP (Contention Period), as shown in Fig. 2. In CFP, AP polls each stream once a polling round, providing a predictable access to each delay sensitive streams. For simplicity, this paper assumes that the polling round coincides with a superframe, but this assumption can be easily generalized. Appropriate time amount is allocated to each multicast stream, and the length of time interval is calculated according to the QoS requirement. The multicast stream is also an instance of message stream that flows from AP to WLAN. On the contrary, during CP any station can send non-real-time message after contending the shared medium via CSMA/CA.

\subsection{Error Model}

WLANs may experience location-dependent channel errors, now that some MSs can correctly communicate with the AP, while at the same time others may suffer packet drops due to errors on the channel. Therefore, given $N$ MSs, there are also $N$ independent error models. An 802.11 radio channel is modeled as a Gilbert channel[8], where two states, good and bad, linked with a Marcov chain, represent the state of channel during an 802.11 slot time. A MAC protocol data unit is received correctly if the channel is in state good for the whole duration of transmission, otherwise, it is received in error. We denote the transition probability from state good to state bad by $p$ and the probability from state bad to state good by $q$. The pair of $p$ and $q$ representing a range of channel conditions, has been obtained by using the trace-based channel estimation. After all, the average error probability, denoted by $\epsilon$, and the average length of a burst of errors are derived as $\frac{p}{p+q}$ and $\frac{1}{q}$, respectively.

\subsection{Message Model}

The video stream traffic occupies the network as a form of streaming-specific packets. For example, H.264 standard decouples the coding aspect from the bit 
stream adaptation needed to transmit over a particular channel such as WLAN. Such multimedia traffic is typically isochronous (or synchronous), consisting of message streams that are generated by their sources on a continuing basis and delivered to their respective destinations also on a continuing basis [9]. The QoS requirement is submitted to $\mathrm{AP}$ and the most important traffic characteristics of each stream are its period and message size. If we assume that there are $n$ multicast video streams in a cell, namely, $S_{1}, S_{2}, \ldots$, and $S_{n}$, each stream can be modeled as follows: A message arrives at the beginning of its period and it must be transmitted by the end of period. The deadline is soft in that some delayed packets are permissible and transmission jitter is absorbed by a playback buffer in the video player. The period of $S_{i}$, is denoted as $P_{i}$, and the maximum length of a message as $C_{i}$.

\section{Error Control Scheme}

\subsection{Bandwidth Allocation}

By allocation, we mean the procedure of determining capacity vector, $\left\{H_{i}\right\}$, for the given superframe time, $F$, and message stream set described as $\left\{S_{i}\left(P_{i}, C_{i}\right)\right\}$. Fig. 2 also illustrates that the slot size is not fixed, namely, $H_{i} \neq H_{j}$ for different $i$ and $j$. At this figure, a message of size up to $C_{i}$ is generated and buffered at regular intervals of $P_{i}$, and then transmitted by $H_{i}$ every time the node is polled. This subsection briefly describes the allocation scheme of Lee's work [5].

Let $\delta$ denote the total overhead of a superframe including polling latency, IFS, exchange of beacon frame, and the like, while $D_{\max }$ the maximum length of a non-real-time data packet. In addition, $P_{\text {min }}$ denotes the smallest element of set $\left\{P_{i}\right\}$. Then the requirement for the superframe time, $F$, can be summarized as in Ineq. (1). Within this range, the scheme can select $F$ and slightly modify $P_{i}$ 's such that they are harmonic [9].

$$
\sum H_{i}+\delta+2 \cdot D_{\max } \leq F \leq P_{\min }
$$

In addition, the least bound of $H_{i}$ that can meet the time constraint of $S_{i}$ is calculated as in Eq. (2).

$$
\begin{array}{ll}
H_{i}=\frac{C_{i}}{\left(\left\lfloor\frac{P_{i}}{F}\right\rfloor-1\right)} & \text { if }\left(P_{i}-\left\lfloor\frac{P_{i}}{F}\right\rfloor \cdot F\right) \leq D_{\max } \\
H_{i}=\frac{C_{i}}{\left\lfloor\frac{P_{i}}{F}\right\rfloor} & \text { Otherwise }
\end{array}
$$

The allocation vector calculated by Eq. (2) is a feasible schedule if the vector meets Ineq. (1). Finally, we can determine the length of CFP $\left(T_{C F P}\right)$ and that of $\mathrm{CP}\left(T_{C P}\right)$ as follows:

$$
T_{C F P}=\sum H_{i}+\delta, \quad T_{C P}=F-T_{C F P} \geq D_{\max }
$$

This scheme is based on the analysis of the worst case available time. Since such an allocation usually considers the upper bound of message size, especially in multimedia applications, unused bandwidth degrades the network throughput. For detailed description of bandwidth allocation, refer to [5]. 


\subsection{Error Report}

A message is divided into packets of size $H_{i}$, hence, for a message to be correctly assembled at a receiver, the receiver should receive all packets successfully. If a packet experiences an error during transmission, additional explicit messages should be sent to request the retransmission of that packet. Per-packet response to the receiver increases overhead, but if the receiver reports the list of damaged packets after it estimates the end of delivery of one message, this waste can be eliminated. It is desirable to send error report via the contention period in order not to interfere the transmission of other stream. If the size of every message is fixed and known in advance, the receiver can easily decide the end of one message transmission. However, the size varies message by message in multimedia streams. Hence, we make all packets include a field specifying the number of packets of the message to which they belong so that the receiver can decide when to report error packet list as long as it receives at least one packet.

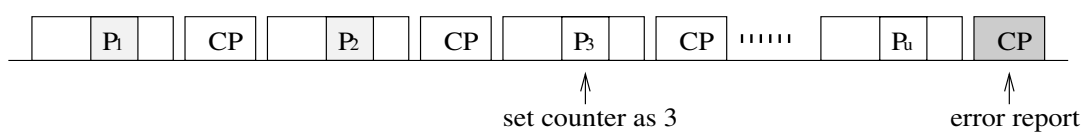

Fig. 3. An example of error reporting

To construct the error report message, the receiver initializes the error packet list when it receives a packet arrives. If the sequence number of this packet is not 1 but $k$, the receiver appends the numbers of 1 through $(k-1)$ to the list. From then, the receiver appends each number of the erroneously received packets. As the receiver also hears Beacon and a stream sends a packet per a polling round, it knows the end of transmission even if the last message is omitted. Fig. 3 shows the example, where a message is transmitted with $u$ packets and each of them has an information that specifies $u$ as well as its sequence number and message identifier. At first, a packet numbered as 3 (not 1) arrives. Then the receiver initializes and adds 1 and 2 to error list as well as sets counter to 3 . The counter increases each time the receiver hears Beacon frame. When the counter reaches $u$, receiver sends error report back to the sender via contention period.

\subsection{Retransmission}

AP receives error report containing error packet list from the receivers of multicast via $\mathrm{CP}$ each time it completes a message transmission until it begins a new transmission. AP builds a retry list sorted by the frequency of appearance in the set of error reports. A new error list arrival reorders the list. Then AP resends the packet according to the order in the retry list when it meets an extra slot for the corresponding video stream. The retransmission scheme can be extended to consider the priority of packet given by the coding scheme performed at the upper layer. 


\section{Performance Analysis}

This section begins with the description of analytic model for the recovered error for the proposed scheme. The average extra bandwidth for a message, $R_{i}$, can be calculated as shown in Eq. (4).

$$
R_{i}=\left(\frac{H_{i}}{F}-\frac{\bar{C}_{i}}{P_{i}}\right) \cdot P_{i}
$$

Then the average number of extra access time, $w$, can be approximated as $R_{i} / H_{i}$. If there are $w$ extra frames for a message composed of $k$ frames, the probability of successful transmission of a message, $T(k, w)$, is calculated recursively. Namely,

$$
T(k, w)=\sum_{i=0}^{m}{ }_{k} C_{i} \cdot \epsilon^{i}(1-\epsilon)^{k-i} \cdot T(i, w-i), T(0, w)=1, T(k, 0)=(1-\epsilon)^{k}
$$

where $m$ is the smaller of $k$ and $w$ while $\epsilon$ denotes the frame error rate.

Now we will show the performance measurement results obtained via simulation using ns-2 [10]. To begin with, we assume that there are $10 \mathrm{MSs}$ in a cell, 3-5 video streams exist simultaneously. Each video stream has the same traffic requirement for simplicity such as bit rate, error characteristic, video packet size, and the number of receivers. Fig. 4 and Fig. 5 plots the average deadline meet ratio observed at each receiver according to BER (Bit Error Rate) and peakaverage ratio. In these figures, the number of messages not packets is counted. The curve denoted as fixed length is for the case where the receiver only knows the maximum length of message. Fig. 4 exhibits that the proposed scheme can reduce the deadline miss ratio by $23 \%$ compared with fixed length scheme and by $48 \%$ compared to no error control case, respectively, when BER is around $10^{-6}$. In Fig. 5, when the peak-average ratio of the message is 1 (all message sizes are equal), both proposed and fixed length schemes show same performance as expected. However, the proposed scheme improves the deadline meet ratio due to the efficient error report mechanism when the ratio increases over 1.0. Other results are omitted due to space limitation. After all, the simulation results demonstrate that overbooked bandwidth can be efficiently used for error recovery of multicast video stream.

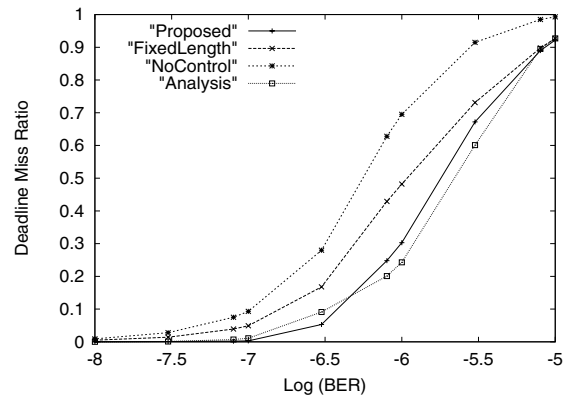

Fig. 4. DMR vs. BER

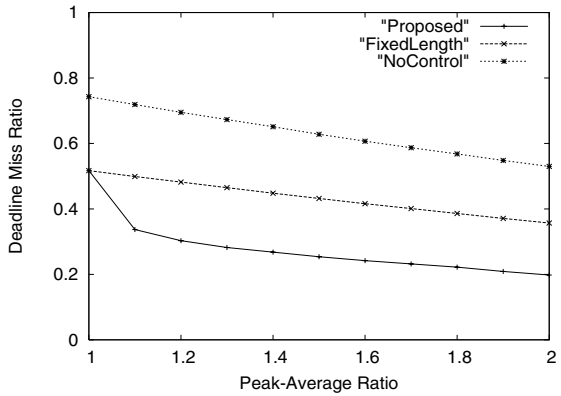

Fig. 5. DMR vs. Peak-average ratio 


\section{Conclusion}

In this paper, we have proposed and analyzed the performance of an error control scheme for multicast video streaming over IEEE 802.11 WLAN. The proposed error control scheme makes the receiver node report errors in a best-effort manner via $\mathrm{CP}$, and also makes AP retransmit the requested packets through the overallocated slot that is unavoidably brought by QoS guarantee in CFP. Therefore, this scheme is able to eliminate the interference to the guaranteed stream transmission, while the message size contained in each frame enables the timely report of error list as long as a packet of message arrives at the receiver. A continuous trace of each video stream channel shows that the proposed scheme can not only efficiently utilize the network bandwidth, but also reduce the deadline miss ratio by $23 \%$ compared with fixed length scheme and by $48 \%$ compared with no error control case, respectively, for the given simulation parameters. As a future work, we are planning to develop a video streaming on dual wireless links focusing on the guarantee scheme and transmission schedule that can efficiently cope with network errors based on the temporarily redundant channel.

\section{References}

1. Mao, S., Lin, S., Wang, Y., Panwar, S. S., Li, Y.: Multipath video transport over wireless ad hoc networks. IEEE Wireless Communications (2005)

2. Majumdar, A., Sachs, D., Kozintsev, I., Ramchandran, K.: Multicast and unicast real-time video streaming over wireless LANs. IEEE Trans. Circuit and Systems for Video Technology, 12 (2002) 524-534

3. Masala, E., Chiasserini, C., Meo, M., De Martin, J.: Real-time transmission of H.264 video over 802.11-based wireless ad hoc networks. Proceedings of Workshop on DSP in Mobile and Vehicular Systems (2003)

4. IEEE 802.11-1999: Part 11: Wireless LAN Medium Access Control (MAC) and Physical Layer (PHY) Specifications (1999) also available at http://standards.ieee.org/getieee802

5. Lee, J., Kang, M., Jin, Y., Kim, H., Kim, J.: An efficient bandwidth management scheme for a hard real-time fuzzy control system based on the wireless LAN. Lecture Notes in Artificial Intelligence, Vol. 3642. Springer-Verlag, Berlin Heidelberg New York (2005) 644-659

6. Shah, S., Chen, K., Nahrstedt, K.: Dynamic Bandwidth Management for Singlehop Ad Hoc Wireless Networks. ACM/Kluwer Mobile Networks and Applications (MONET) Journal. 10 (2005) 199-217

7. Lu, A., Chen, T., Steenkiste, P.: Video Streaming over 802.11 WLAN with Contextaware Adaptive Retry. IEEE International Conference on Multimedia and Expo (2005)

8. Bottigliengo, M., Casetti, C., Chiaserini, C., Meo, M.: Short term fairness for TCP flows in 802.11b WLANs. Proc. IEEE INFOCOM (2004)

9. Carley, T., Ba, M., Barua, R., Stewart, D.: Contention-free periodic message scheduler medium access control in wireless sensor/actuator networks. IEEE Real-Time Systems Symposium (2003)

10. Fall, K., Varadhan, K.: Ns notes and documentation. Technical Report, VINT project, UC-Berkeley and LBNL (1997) 